\title{
Intralobar Bronchopulmonary Sequestration: A Case and Brief Review
}

\author{
Uddalak Majumdar, MD' \\ Payal Sen, $M D^{2}$ \\ Akshay Sood, $M D^{2}$ \\ ${ }^{1}$ Cleveland Clinic Foundation, Cleveland, OH USA \\ 2Univeristy of New Mexico, Albuquerque, NM USA
}

\begin{abstract}
Objective: Bronchopulmonary sequestration is a rare congenital abnormality of the lower respiratory tract, seen mostly in children but often in adults. The term implies a mass of lung tissue that has no function and lacks normal communication with the rest of the tracheobronchial tree.

Case: A 40-year-old man presented with acute onset of left flank pain for 4 hours. He was born in Yemen and emigrated to the US in 1998; at that time, he had been tested for tuberculosis which was negative. In this admission, he met systemic inflammatory response (SIRS) criteria and had basilar crackles in the left lower lobe of the lung. CT scan revealed a cavitary lesion with air-fluid level in the left lower lobe airspace. There was systemic arterial blood supply to this region arising off the celiac axis. He was diagnosed with an infected intralobar bronchopulmonary sequestration and underwent video-assisted thoracoscopic wedge resection. On follow up 3 months later, he was doing well.

Discussion: Pulmonary sequestration is a rare congenital anomaly of a mass of lung tissue, which can have cystic changes and is a very important differential diagnosis of cavities in the lung. Confirmation of diagnosis is by visualization of a systemic vessel supplying sequestrated pulmonary, and this is accomplished by contrast-enhanced CT scan, MRI or invasive angiography.

Conclusion: The delay in diagnosis in our patient was due to falling prey to anchoring and availability biases and chasing the diagnosis of tuberculosis in a patient from Yemen with a lower lobe cavitation.

Case

History of Present Illness: A 40-year-old man with a past medical history of atrial fibrillation presented to the hospital with acute onset of left flank pain for 4 hours, fevers and chills. The pain was sharp and stabbing, pleuritic, non-radiating, and was severe with an intensity of 10/10. He denied extraneous activity or trauma earlier in the day, denied substernal pain, cough, night sweats, weight loss or change in urinary habits. He was born in Yemen and emigrated to the US in 1998; at that time, he was tested for tuberculosis (TB) which was negative. He was known to have a cavitary lesion in left lower lobe since 2005, and had undergone extensive evaluation (imaging, sputum and PPD) which showed no form of tuberculosis. He denied taking prophylactic TB treatment. Annual PPD testing had always been negative.
\end{abstract}


The patient worked on a ship, which travelled in the Great Lakes on the US-Canada border. He was a current smoker with a 20-pack-year smoking history. He lived at home with his wife and children. There was no history of IV drug use, prior imprisonment or homelessness. He denied being in contact with anyone with TB while in Yemen. He was sexually active with his wife and had no other sexual partners. He denied history of sexually transmitted infections.

\section{Physical Examination:}

Vital Signs: Temp - 38.3 degrees Fahrenheit, Pulse- 111/minute, RR- 18/min, BP151/66 mm Hg. Spo2- $90 \%$ on Room Air.

Basilar crackles and rhonchi in the left lower lobe of the lung. No cervical or inguinal lymphadenopathy. Rest of the physical exam was normal.

\section{Significant Laboratory Findings:}

WBC elevated at $15,500 / \mathrm{mm}^{3}$ with 65 percent Neutrophils.

Lactate $-1.1 \mathrm{mmol} / \mathrm{dL}$

\section{Radiography:}

Chest $\mathrm{x}$-ray was done while in the emergency department, which revealed left basilar sub-segmental atelectasis (Figure 1).

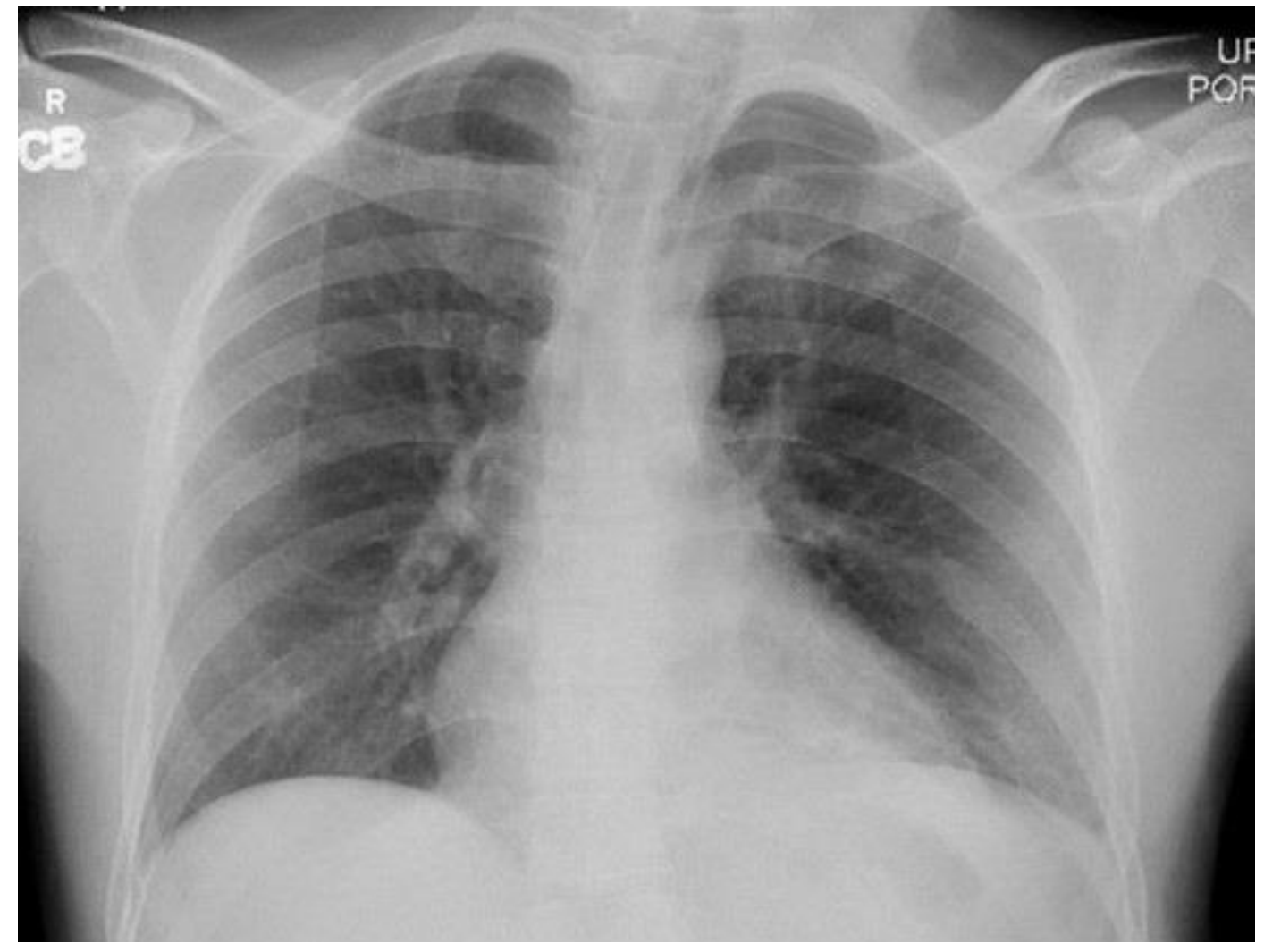

Fig.1. Chest $x$-ray showing left basilar sub-segmental atelectasis without focal consolidation, large pleural effusion or pneumothorax. 
Initial CT scan of abdomen and pelvis was done to rule out renal/ureteral stone. It showed a left lower lobe airspace consolidation with bronchiectasis and bronchiolectasis and a cavitary lesion with air-fluid level (Figure 2).

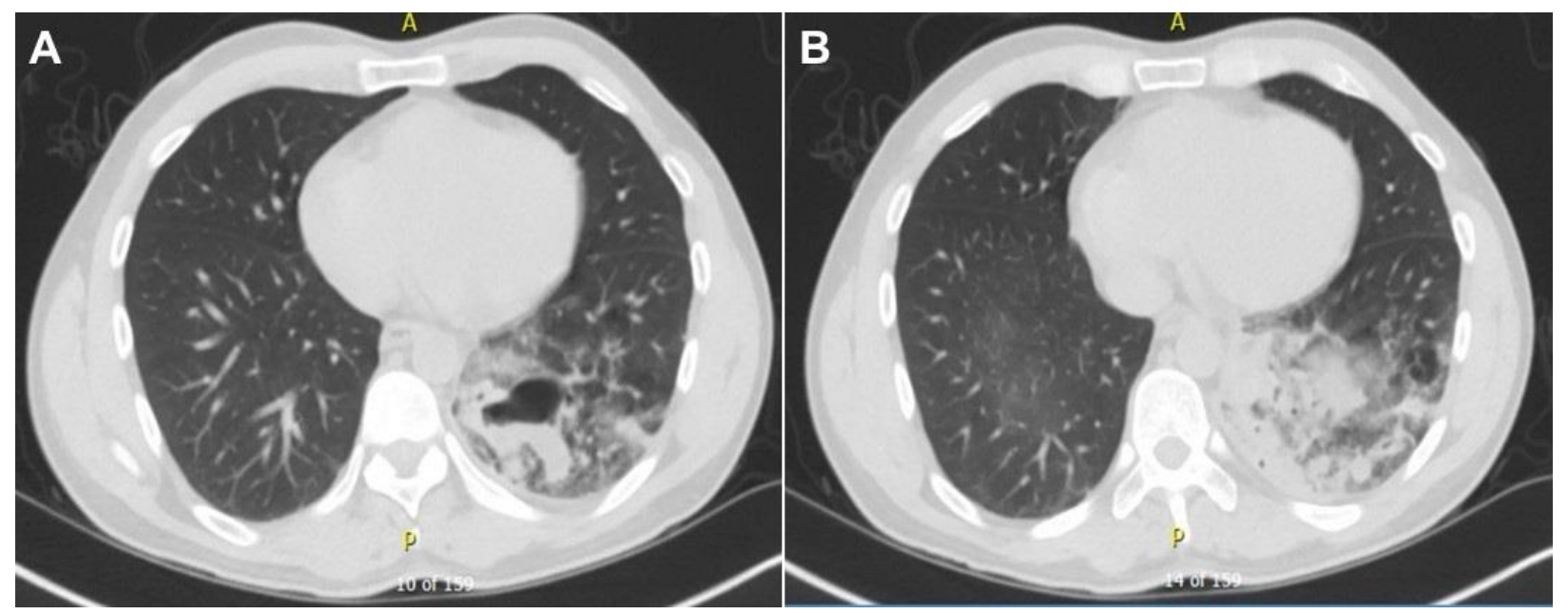

Figure 2. Representative images from the CT scan in lung windows showing left lower lobe airspace consolidation concerning for an acute on chronic process.

C-reactive protein and erythrocyte sedimentation rate were normal, CRP and ESRnormal; blood cultures revealed no growth; procalcitonin $0.4 \mathrm{ng} / \mathrm{mL}$ (normal $<0.15$ ); anti-nuclear antibody - negative; Aspergillus antigen - negative; urine Legionella antigen - negative; Streptococcus pneumoniae antigen - positive.

Sputum Gram stain and acid-fast bacilli culture/stain could not be obtained because the patient did not produce any sputum.

Subsequently CT chest with IV contrast was done which showed findings compatible with a pneumonia within a left lower lobe intrapulmonary sequestration. (Figure 3).
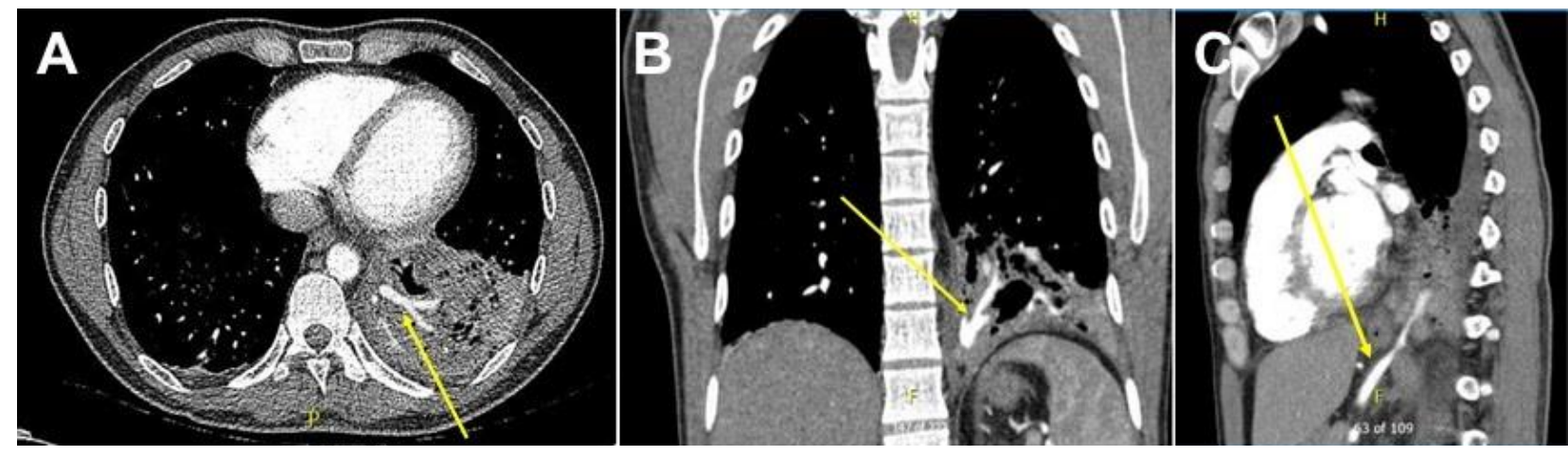

Figure 3. Representative images from the thoracic CT chest with IV contrast. The left lower lobe demonstrates a $69 \times 83 \mathrm{~mm}$ heterogeneous fluid collection with multiple locules of air. There was systemic arterial blood supply to this region arising off the celiac axis (arrows). 
The patient was diagnosed with an infected intralobar bronchopulmonary sequestration. He was treated initially with intravenous fluids and piperacillintazobactam. He underwent video-assisted thoracoscopic wedge resection of infected bronchopulmonary sequestration in left lower lobe and ligation of the systemic feeding vessels from the celiac artery. Pathologic examination revealed a fibrotic lung with areas of centrilobular emphysema, bronchiolectasis, mucus pooling and microscopic honeycomb changes. Findings also showed an elastic artery, with features most suggestive of intralobar sequestration. His symptoms completely resolved after his operation.

\section{Discussion}

Bronchopulmonary sequestration is a rare congenital abnormality of the lower respiratory tract, seen mostly in children but often in adults, like in our patient (1). In 1946, Pryce coined the term "pulmonary sequestration" to describe a disconnected bronchopulmonary mass or cyst with an anomalous arterial supply (2). The term implies a mass of lung tissue that has no function and lacks normal communication with the rest of the tracheobronchial tree. This mass of non-functional lung tissue receives blood supply from the systemic circulation (3). The exact etiology is unknown and is thought to be an embryologic process error in foregut budding (4), although some have indicated a non-congenital acquired process in intralobar sequestration.

Sequestration may be intra- or extralobar based on its relation with the normal lung lobes. An intralobar sequestration (ILS), like the name suggests, is located within a normal lobe, lacks its own visceral pleura (5) and also has aberrant connections to bronchi, and lung parenchyma, or even the gastrointestinal tract, and often presents with recurrent infections $(6,7)$. Compared to ILS, an extralobar sequestration (ELS) is located outside the normal lung and has its own visceral pleura (8), with the rare occurrence of infectious complications (9). About $75 \%$ of BPS is intralobar while $25 \%$ is extralobar (10). Bronchopulmonary sequestration is often associated with other congenital abnormalities like congenital diaphragmatic hernia, vertebral anomalies, congenital heart disease, pulmonary hypoplasia, colonic duplication, and congenital pulmonary airway malformation (11).

Clinically, pulmonary sequestration is latent until infection leads to symptoms (12). Symptoms, like that of any pathological lung condition depend on the type, size, and location of the lesion. Sepsis and extracardiac shunting are common complications of untreated sequestration. Hemoptysis can also be a presentation. The mechanism of pneumonia is post-obstructive and usually recurrence of pneumonia leads to diagnosis. Recurrent pneumonia especially in the lower lobes should always include intralobar sequestration in the differential diagnoses. But the pathophysiology of infection and/or hemoptysis when ILS is not connected to airway is a mystery. Sometimes there is a partial or anatomically abnormal connection to the tracheobronchial tree, which can lead to poor mucus clearance, plugging and recurrent infection. 
The mainstay of diagnosis is pre-operative imaging and post-operative histopathology of the resected specimen. The pathognomonic imaging characteristic is systemic vascular supply of the affected area of the lung (intra or extra-lobar), which is seen in about $80 \%$ of CT scans. Recurrent infection can lead to cystic areas within the mass (clusters of "ring shadows" on X-ray) (13). The surrounding normal lung may have air trapping and show emphysematous changes. Radiologic signs of BPS are a spectrum and represent the chronic and recurrent inflammation of the sequestrated lung: recurrent focal airspace disease, a parenchymal mass, a cavitary consolidation or mass, cystic lesions, localized bronchiectasis or adjacent emphysema. Bronchoscopy has little role in the management of BPS, which needs to be kept in mind by clinicians investigating cystic lung lesions. Identifying the systemic feeding vessel also helps with surgical planning.

Symptomatic patients are treated with surgical excision; surgery is curative and is associated with minimal morbidity (14). Surgery is urgent in patients with significant respiratory distress but may be an elective procedure in adults or older children with less symptoms $(15,16)$.

For asymptomatic patients of any age, management depends on how 'high risk' they are considered for developing complications. High risk patients are those with large lesions occupying $>20$ percent of the hemithorax, bilateral or multifocal cysts, or those with pneumothorax. In these patients, surgical resection is preferred to observation (17). On the other hand, in asymptomatic patients without these high-risk characteristics, either elective surgical resection or conservative management with observation are reasonable options (18).

Apart from surgery, even embolization of the anomalous arterial supply has been reported to result in a complete resolution of symptoms and imaging changes to a certain in some cases (19). Since identification of vascular supply during surgery may be difficult during surgery, presurgical embolization may reduce risk of vascular complications (19). Embolization also has a more important role in hemoptysis and heart failure from shunting.

\section{Conclusions}

- Pulmonary sequestration is a rare congenital anomaly of a mass of lung tissue without a normal connection to the tracheobronchial tree and a systemic vascular supply.

- Presentation in adults is due to complication of the mass, undiagnosed in childhood.

- Sequestrated lung can have cystic changes and is a very important differential diagnosis of the cavitation.

- Confirmation of diagnosis is by visualization of a systemic vessel supplying sequestrated pulmonary, and this is usually accomplished by contrast-enhanced CT scan, MRI or invasive angiography. 


\section{Teaching points}

This is a case of adult presentation of congenital pulmonary malformation and represents a delay in diagnosis, even though the patient's symptoms started 10 years ago. The delay was due to falling prey to anchoring and availability biases and chasing the diagnosis of TB ten years ago in a patient from Yemen with a lower lobe cavitation. The feeding vessel from the celiac axis can only be demonstrated via a contrast enhanced CT, and thus, when in doubt, we should always get angiography by contrastenhanced-CT or MRI or by invasive angiography. Had it been thought of and done 10 years ago, the patient would've been diagnosed and treated earlier.

\section{Disclosure statement}

Drs. Majumdar, Sen and Sood have no conflicts of interest or financial ties to disclose.

\section{References}

1. Landing BH, Dixon LG. Congenital malformations and genetic disorders of the respiratory tract (larynx, trachea, bronchi, and lungs). Am Rev Respir Dis. 1979 Jul;120(1):151-85. [CrossRef] [PubMed]

2. John PR, Beasley SW, Mayne V. Pulmonary sequestration and related congenital disorders. A clinico-radiological review of 41 cases. Pediatric radiology. Pediatr Radiol. 1989;20(1-2):4-9. [CrossRef] [PubMed]

3. Van Raemdonck D, De Boeck K, Devlieger $\mathrm{H}$, et al. Pulmonary sequestration: a comparison between pediatric and adult patients. Eur J Cardiothorac Surg. 2001 Apr;19(4):388-95. [CrossRef] [PubMed]

4. Gezer S, Taştepe I, Sirmali M, Findik G, Türüt H, Kaya S, Karaoğlanoğlu N, Cetin G. Pulmonary sequestration: a single-institutional series composed of 27 cases. J Thorac Cardiovasc Surg. 2007 Apr;133(4):955-9. [CrossRef] [PubMed]

5. Shanti CM, Klein MD. Cystic lung disease. Semin Pediatr Surg. 2008 Feb;17(1):2-8. [CrossRef] [PubMed]

6. Stocker JT, Drake RM, Madewell JE. Cystic and congenital lung disease in the newborn. Perspect Pediatr Pathol. 1978;4:93-154. [PubMed]

7. Schwartz MZ, Ramachandran P. Congenital malformations of the lung and mediastinum--a quarter century of experience from a single institution. J Pediatr Surg. 1997 Jan;32(1):44-7. [CrossRef] [PubMed]

8. Abbey P, Das CJ, Pangtey GS, Seith A, Dutta R, Kumar A. Imaging in bronchopulmonary sequestration. Send to J Med Imaging Radiat Oncol. 2009 Feb;53(1):22-31. [CrossRef] [PubMed]

9. Houda el M, Ahmed Z, Amine K, Amina BS, Raja F, Chiraz H. Antenatal diagnosis of extralobar pulmonary sequestration. Pan Afr Med J. 2014;19:54. [CrossRef] [PubMed]

10. Frazier AA, Rosado de Christenson ML, Stocker JT, Templeton PA. Intralobar sequestration: radiologic-pathologic correlation. Radiographics. 1997 MayJun;17(3):725-45. [CrossRef] [PubMed] 
11. Kravitz RM. Congenital malformations of the lung. Pediatr Clin North Am. 1994 Jun;41(3):453-72. [CrossRef] [PubMed]

12. Hang JD, Guo QY, Chen CX, Chen LY. Imaging approach to the diagnosis of pulmonary sequestration. Acta Radiol. 1996 Nov;37(6):883-8. [CrossRef] [PubMed]

13. Hernanz-Schulman M. Cysts and cystlike lesions of the lung. Radiol Clin North Am. 1993 May;31(3):631-49. [PubMed]

14. Samuel M, Burge DM. Management of antenatally diagnosed pulmonary sequestration associated with congenital cystic adenomatoid malformation. Thorax. 1999 Aug;54(8):701-6. [CrossRef] [PubMed]

15. Haller JA, Jr., Golladay ES, Pickard LR, Tepas JJ, 3rd, Shorter NA, Shermeta DW. Surgical management of lung bud anomalies: lobar emphysema, bronchogenic cyst, cystic adenomatoid malformation, and intralobar pulmonary sequestration. Ann Thorac Surg. 1979 Jul;28(1):33-43. [CrossRef] [PubMed]

16. Al-Bassam A, Al-Rabeeah A, Al-Nassar S, Al-Mobaireek K, Al-Rawaf A, Banjer $\mathrm{H}$, et al. Congenital cystic disease of the lung in infants and children (experience with 57 cases). Eur J Pediatr Surg. 1999 Dec;9(6):364-8. [CrossRef] [PubMed]

17. Parikh DH, Rasiah SV. Congenital lung lesions: Postnatal management and outcome. Semin Pediatr Surg. 2015 Aug;24(4):160-7. [CrossRef] [PubMed]

18. Singh $\mathrm{R}$, Davenport $M$. The argument for operative approach to asymptomatic lung lesions. Semin Pediatr Surg. 2015 Aug;24(4):187-95. [CrossRef] [PubMed]

19. Eber E. Adult outcome of congenital lower respiratory tract malformations. Swiss Med Wkly. 2006 Apr 15;136(15-16):233-40. [PubMed] 